$>$ Rassembler des documents d'archives pour retracer l'histoire de la compagnie Zeiss ne présente aucune difficulté : ils abondent... sauf pour une période allant de 1932 à 1945, systématiquement passée sous silence et qui correspond précisément à la période nazie. Sur le site internet Zeiss historica, parmi les personnalités marquantes de la compagnie Zeiss, on note que, pour Emanuel Goldberg, la page est «still under development but an early picture of the professor is available». A partir de cette indication, tout s'éclaire. Car, si la compagnie Zeiss n'a pas apporté de précisions sur cette «personnalité marquante », les travaux d'un chercheur de la School of information management and systems de l'Université de Californie, Mickael Buckland, nous mettent sur la voie. Grâce à lui, le nom et les travaux de Goldberg, qui avaient disparu de notre héritage culturel et scientifique, reviennent en lumière après cinquante ans d'effacement. Goldberg avait pourtant publié des dizaines d'articles, obtenu des brevets, mis au point des appareils de photographie, de cinéma, et surtout il avait été l'inventeur du premier support électronique de recueil et d'accès aux connaissances. $\varepsilon$ Erance, si cette redécouverte a été portée à la connaissance du monde des sciences de l'information, elle n'a pas eu le retentissement qu'elle méritait dans le monde scientifique. C'est pourquoi le temps est venu de retracer le parcours de cet ingénieur hors pair, et d'expliquer les raisons qui ont gommé de l'Histoire son nom et sa mémoire. <

\section{Grandeur et vicissitudes de deux grandes compagnies d'optique allemandes}

\section{Zeiss Ikon et I'élimination d'Emanuel Goldberg}

Simone Gilgenkrantz

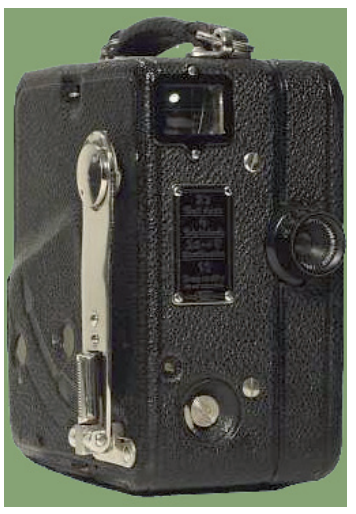

S. Gilgenkrantz :

9 , rue Basse, 54330 Clérey-sur-Brénon, France. simsimone.gilgenkrantz@ gmail.com

\section{Le professeur Goldberg, directeur de Zeiss Ikon}

En 1917, le professeur Goldberg (1881-1970) est directeur de Ica, I'Internationale camera Atkiengesellschaft à Dresde, capitale de l'État de Saxe et important centre industriel de production photographique.

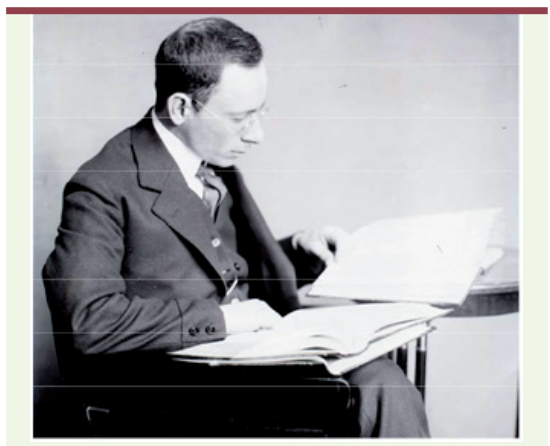

Emanuel Goldberg chez Zeiss Ikon vers 1924

En 1920, Ica s'associe avec Contessa-Nettel (Stuttgart). À cette époque, malgré la terrible inflation qui sévit en Allemagne, l'exportation de matériel aide les firmes à se maintenir. Pourtant, en 1926, en raison de difficultés financières, la Carl Zeiss Stiftung rachète Ica/Contessa, plus Ernemann (Dresde) et Goetz (Berlin), deux autres manufactu- 
res d'optique, pour former la Zeiss Ikon AG. Cette «fusion», terme diplomatique, en fait la plus grande compagnie allemande de matériel photographique.

Zeiss Ikon obtient alors de nombreux brevets, et devant le succès du Leica de chez Leitz [1, 2], Goldberg fabrique un appareil photo, le Contax I. II comporte des innovations intéressantes, parmi lesquelles un système à baïonnette pour fixer l'objectif, un changement plus facile des pellicules, une vitesse d'obturation supérieure.

En 1931, le huitième Congrès international de photographie a lieu à Dresde. Une chance pour Goldberg qui avait déjà participé aux congrès antérieurs. II joue un rôle majeur dans l'organisation de celui-ci, qui rassemble plus de 400 participants. À cette occasion, des efforts sont faits pour standardiser le matériel, et des accords sont passés avec la France et les États-Unis. Outre les publications techniques sur la photographie et le cinéma (contrôle sensitométrique de l'exposition, photogravure, caractéristique des émulsions), Goldberg fait aussi une démonstration grand public : à partir de l'enregistrement de l'ouverture d'Egmont de Beethoven, il démontre que les vibrations sonores de la musique peuvent être converties en courant électrique puis, à l'aide d'une cellule photoélectrique, restituées dans un haut parleur. Au banquet final, il est décoré de la médaille Péligot, décernée pour la première fois à un étranger par le professeur Léopold Lobel au nom de la Société française de photo et de cinématographie. II ignore alors qu'il est parvenu au faîte de sa carrière.

\section{Les SA (Sturm Abteilung : sections d'assaut)}

Le 6 mars 1933, Fritz Busch, directeur renommé de l'opéra Semper de Dresde revient de Berlin par le train avec le chef d'orchestre 0tto Klemperer. Tous deux, notoirement antinazis, s'insurgent à voix haute de la prise de pouvoir de Hitler et se lamentent sur l'avenir de l'Allemagne. Ils ignorent que la personne qui se trouve en face d'eux est le nouveau gouverneur de Saxe, Manfred von Killinger, ancien héros de la Grande guerre, tout récemment nommé ministerpräsident par le Führer. Le lendemain, à l'opéra, la représentation de Rigoletto est brutalement interrompue par une soixantaine de SA en armes. Ils exigent la démission de Busch, celui-ci n'ayant pas obtempéré à l'ordre de chasser les musiciens et chanteurs juifs du Semperoper.

Le 15 mars, le maire de Dresde est destitué et le parti nazi prend officiellement le pouvoir en Saxe. Les communistes ont été arrêtés et les locaux du journal de gauche envahis et brûlés.

Le $1^{\text {er }}$ avril 1933, Hitler proclame le boycott national de tous les magasins juifs. Goldberg reçoit un appel d'un responsable de la firme Goetz à Berlin le prévenant de la demande d'exclusion de tous les employés juifs de Zeiss Ikon. Comme c'est un samedi, l'usine est fermée. Les responsables se réunissent et préviennent le siège de la Carl Zeiss Stiftung à léna.

Le lundi 3 avril, des tracts sont distribués aux 220 employés de Zeiss Ikon leur demandant de venir à la réunion générale. Un peu plus tard, des SA accompagnés de quelques employés pénètrent dans le bureau du directeur commercial Alfred Simader. Revolver au poing, ils emmènent de force Goldberg et Grentz, sans que les autres respon- sables présents puissent intervenir. Dehors, ils veulent contraindre le chauffeur de la voiture de fonction de les conduire, mais en dépit des menaces, celui-ci refuse catégoriquement. Ils s'emparent donc de la Mercedes de Goldberg et disparaissent avec leurs captifs.

Les responsables, Ernemann et Simader, finissent par joindre le gouverneur von Killinger. De toute évidence, celui-ci n'est pas au courant. II les assure de son soutien. Quelques jours plus tard paraîtront deux articles dans les journaux : I'un émanant de von Killinger accusant «des éléments incontrôlés », l'autre du Gauleiter Mutschmann, précisant que «la lutte continue, mais que toutes les initiatives individuelles sont interdites $\gg$.

Le 6 avril à 3 heures du matin, après avoir dû écrire une lettre de démission, Emanuel Goldberg est libéré. II rentre chez lui, choqué, mais vivant.

Avant de connaître la suite de sa carrière après cet enlèvement, revenons d'abord sur ses débuts.

\section{Les origines}

Emanuel Goldberg est né à Moscou en 1881. Son père était colonel dans le corps de santé de l'armée du Tsar. Pour sa participation à la guerre russo-turque (187778), il avait été couvert de médailles et, fait assez rare pour un juif, il avait même reçu la médaille de Saint Stanislas ${ }^{1}$ et avait le titre de conseiller à la cour. Élève brillant, passionné de physique et de chimie, Emanuel espère entrer à l'École technique impériale. Mais malgré des notes suffisantes, le numerus clausus imposé aux juifs (3\% seulement des admis) fait qu'il est refusé. À l'Université de Moscou, il obtient un diplôme de chimie, puis il va en Allemagne, s'inscrit à l'Université de Leipzig où il passe son doctorat en 1906, avec une thèse sur la cinétique des réactions photochimiques. Son professeur, Robert Luther, alors sous-directeur de I'Institut Wilhelm Ostwald ${ }^{2}$ de chimie-physique, lui sert de mentor [3]. Il fait des stages à Moscou, à Londres où il se lie avec Kenneth Mees, jeune étudiant anglais qui deviendra plus tard directeur de recherche à la Eastman Kodak company à Rochester (Ny, États-Unis). Après un an passé comme assistant au Laboratoire de photochimie de l'Université de Berlin, il retourne à Leipzig où il est nommé professeur au département de photographie de l'Académie royale des arts graphiques.

Ce furent sans doute les années les plus heureuses de sa vie $[4,5]$.

\footnotetext{
${ }^{1}$ Médaille portant l'aigle royal pour les non chrétiens et le sigle de Saint Stanislas pour les chrétiens.

${ }^{2}$ Wilhelm Ostwald eut le Prix Nobel de chimie en 1909.
} 
Il a fait la connaissance de la sœur d'un de ses camarades d'étude, Sophie Posniak. De quelques années plus jeune que lui, elle suit des cours de musique et appartient à une riche famille de commerçants juifs de Nijni Novgorod, plutôt sionistes. Leur mariage, en 1907, ne réjouit aucune des deux familles, les Goldberg estimant que les Posniak ne sont pas cultivés, les Posniak trouvant que ce jeune homme manque de fortune et d'élégance.

\section{Ballons captifs et cinéma}

En 1914, quand éclate la guerre, Sophie attend son premier enfant. Le professeur Luther, avec lequel Emanuel Goldberg a gardé des relations, s'engage dans l'armée et devient officier d'un bataillon d'aérostiers. Il demande à Emanuel d'améliorer les appareils photos des drachen, ballons captifs utilisés pour la reconnaissance aérienne. Goldberg modifie leur distance focale et leur stabilité. II prend lui-même des photos aériennes des tranchées ennemies et ces explorations aériennes au-dessus des lignes de front, très dangereuses, inquiètent beaucoup Sophie. Dès 1916, il est contacté par la maison Zeiss pour travailler sur du matériel d'optique pour l'armée.

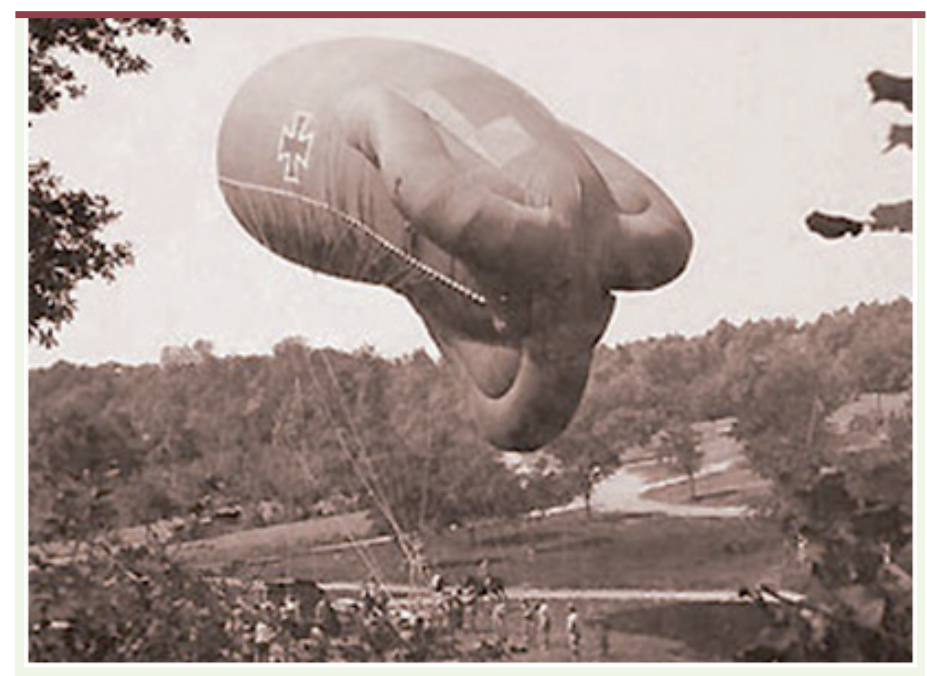

Drachen : ballon cerf-volant allemand de 1916

Chez Ica, où il entre en 1917, il s'applique à moderniser la production. II acquiert des machines-outils de pointe et fabrique de nouveaux équipements photographiques (agrandisseurs, appareils photos, densitomètres, etc.). Le cinéma en est encore à ses débuts et Goldberg crée en 1920 une division cinéma en commençant par fabriquer une caméra $35 \mathrm{~mm}$ qu'il a entièrement conçue : le Kinamo. Cet appareil, très maniable, est parfait pour les films d'amateurs. Goldberg en réalise lui-même, en famille, surtout pendant les vacances d'hiver dans les Dolomites. Mais le Kinamo est plus que cela puisqu'il retient l'attention du cinéaste Joris Ivens qui s'en servira pour tourner et l'évoquera plus tard dans ses mémoires $[6,7]$ : « un homme me fit grande impression, le professeur Goldberg. Il avait inventé une formidable petite caméra, le fameux Kinamo... d'une robustesse et d'une précision ahurissantes pour son temps ».

\section{Microphotos et gestion électronique des connaissances}

À Paris, au sixième Congrès international de la photographie en 1925, pour commémorer le centenaire de sa découverte, Goldberg distribue aux participants des lames de microscope. Elles portent un micropoint dans lequel se trouve la photo de Nicéphore Niépce entouré d'un cercle gradué, de l'ordre d'un centième de $\mathrm{mm}^{2}$. Baptisée le Mikrat, cette microphoto - ingénieuse utilisation du microscope comme caméra - pouvait servir à photographier des textes, des livres, sous une forme extrêmement réduite. Le Mikrat fit l'objet de publications aussi bien en français qu'en allemand et en anglais.

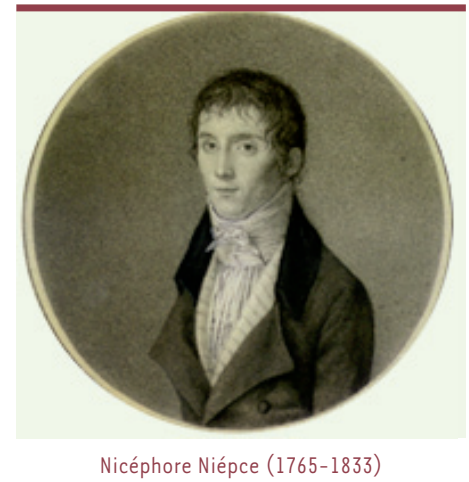

À cette époque, Emanuel Goldberg s'est installé à Dresde. Après leur fils, Herbert, né en 1914, ils auront une fille, Renate, en 1922. Ils habitent dans une jolie maison à trois étages où les parents de Sophie, fuyant la révolution russe, sont venus les rejoindre. Emanuel y a une pièce avec ses machines-outils, interdite aux enfants. II se décrit lui-même comme chimiste de formation, perçu comme physicien, mais profondément mécanicien, capable de travailler aussi volontiers le fer, le bois que le verre ou le diamant, pour réaliser des prototypes. II fait aussi construire une petite maison d'été à Loschwitz, sur la colline, avec une jolie vue de Dresde depuis la rive droite de l'Elbe.

À partir du recueil des données en microphotos se pose un problème : comment fait-on pour les retrouver aisément? II entreprend alors un travail ambitieux : le classement des données, leur conservation et leur reconnaissance à l'aide d'une cellule photoélectrique, capable de lire les codes d'enregistrement. II réalise une sorte de bureau, l'ancêtre d'Internet en quelque sorte. L'appareil est breveté, sa description est publiée en plusieurs langues [8]. En octobre 1931, à la Royal photographic society de Londres, il présente ce qu'il appelle «la Machine Statistique », dans un exposé 
intitulé « La cellule photoélectrique en photographie » qui déclenche l'enthousiasme de l'auditoire. Mais sa notoriété, nous l'avons vu, va être de courte durée.

\section{Le retour aux sources}

Après son enlèvement en 1933, il doit évidemment quitter l'Allemagne. II va à léna où les responsables de Zeiss redoutent qu'à l'étranger, il travaille pour une firme concurrente. Une solution est trouvée: on le nomme responsable dans les succursales de Zeiss France, Ikonta et Optica.

Avant de rejoindre la famille, son fils Herbert aide son collaborateur et ami Simader à déménager la maison. Le superbe piano à queue Blüthner de Sophie est échangé contre un piano droit. Toutes les machines-outils sont empaquetées et envoyées en France. La famille s'installe à Paris et retrouve quelques amis. Mais Goldberg s'ennuie : son activité est purement commerciale et il a d'autres projets en tête. Pour s'occuper, il construit une petite maison d'été, sans électricité, à Chenevières, dans le val de Marne. II la baptise «Une Étape », preuve qu'il sent que son séjour en France ne sera que temporaire. En 1937, il fait un saut en Palestine, y rencontre Chaïm Weizman qui a, lui aussi, étudié la chimie en Allemagne. C'est là qu'il décide de la destination de la dernière étape de sa vie.

II aurait pu choisir les États-Unis. Plusieurs propositions lui étaient faites, en particulier chez Kodak où Kenneth Mees, avec lequel il avait gardé des relations, l'avait invité à plusieurs reprises. Mais la Palestine lui semble «le seul pays où un Juif n'ait pas à souffrir d'antisémitisme ». Herbert, quant à lui, âgé de 22 ans et diplômé de Supelec, décide de tenter sa chance dans le Nouveau Monde.

Avant de partir, Emanuel Goldberg doit encore entamer une lutte âpre et douloureuse avec Zeiss Ikon qui n'accepte sa démission qu'à condition qu'il signe une clause de non-concurrence à vie : il lui serait interdit de travailler dans tous ses domaines de compétence, même sur la «Machine Statistique».

Après de pénibles arrangements, il s'installe enfin en Palestine avec Sophie et sa fille Renate. Le nouveau «Laboratoire pour instruments de précision » qu'il crée à Tel Aviv deviendra le Israeli electro-optical industry. Pendant la période de guerre, il fournit du matériel aux autorités britanniques, conçoit un stylo-caméra, le Ktina, pour l'Intelligence service. Puis, avant que le Royaume-Uni renonce à son mandat sur la Palestine, il aidera aussi la Haganah dont les agents manqueront parfois de croiser, dans ses bureaux, les officiers britanniques.

Mais désormais, et jusqu'à sa mort sur cette terre devenue Israël, plus personne n'entendra parler de lui.

\section{Une refondation de la mémoire}

En 1988, en recherchant les travaux faits en Europe sur les sciences de l'information avant la Seconde Guerre mondiale, Michael Buckland constate que les travaux d'Emanuel Goldberg sont victimes d'une sorte de conspiration du silence. Il entreprend alors une recherche patiente de près d'une vingtaine d'années $[9,10]$ au cours desquelles il reconstitue la vie et la carrière de cet inventeur, et surtout, il identifie les acteurs de cette volonté d'effacement:

- Pour la compagnie Zeiss, le blackout prolongé est d'une implacable logique: dès son départ en 1933, Küppenbender, que nous avons déjà évoqué [11], occupe la place de Goldberg. Puis il prend la direction de l'ensemble de la firme Carl-Zeiss-Stiftung. Après la guerre, il assure son transfert à l'Ouest et contrôle pendant 45 ans les archives présentées dans Zeiss historica.

- Comme inventeur du Mikrat, Goldberg aurait pu être mentionné par les amateurs d'espionnage. Mais un article du Reader's Digest est venu très tôt brouiller les pistes [12]. L'auteur, J. Edgar Hoover, le tout- puissant directeur du Federal Bureau of Investigation, parle effectivement des micropoints, mais - étrangement - il substitue au nom de Goldberg celui d'un certain professeur Zapp de Dresde. Or, le professeur Zapp n'a jamais existé. Erreur ou manipulation volontaire? Car à l'heure de la guerre froide, le FBI n'ignorait rien des outils des espions et de leurs concepteurs. Mais depuis, ce sera toujours le nom de Zapp qui sera cité, même dans les publications les plus récentes [13].

- Enfin, pour tous les Américains, le grand homme de l'intelligence artificielle, le père d'Internet et de l'hypertexte, se nomme Vannevar Bush. Sa publication, dans Atlantic Monthly, en 1945, d'un texte visionnaire: As we may think, annonçait l'ère de l'informatique en même temps que celle de la paix [14]. Elle tombait à pic, à la fin de la Seconde Guerre mondiale, venant d'un homme qui avait été le maître de la recherche scientifique des États-Unis et du « succès » de la bombe atomique. Dans son très long texte, peu lu en France, il y décrit sa découverte, le Memex. Cet extenseur de mémoire (Memory extender) serait un appareil électronique, contenant un système capable d'afficher des textes microfilmés, reliés entre eux. II ne cite aucune référence, alors que les travaux antérieurs ne manquaient pas [15]. Mais surtout, quand il essaie d'obtenir un brevet, on le lui refuse, car un brevet analogue existe déjà, celui de Goldberg datant de 1931. Pourtant, durant le reste de sa vie, Bush continuera à accepter l'adulation du public, sans jamais avouer qu'il avait eu un précurseur.

Les faits sont têtus, malgré tout. Grâce à Mickael Buckland, la vie de Goldberg, ses travaux, sont à présent connus aux États-Unis [10]. Il est temps de les porter à la connaissance du monde scientifique en France. $\diamond$ 


\section{SUMMARY}

Greatness and tribulations of Zeiss and Leitz, two famous German optic companies. III. Zeiss Ikon and elimination of Emanuel

\section{Goldberg}

Gathering archival documents to trace the history of the Zeiss company presents no difficulty : they are abundant... except for a period from 1932 to 1945, systematically ignored, and that corresponds to the Nazi period. On the website Zeiss Historica, among the outstanding personalities of the Zeiss company, we note that, for Professor Emanuel Goldberg, the web page "is still under development but an early picture of the professor is available. ». But fortunately, Mickael Buckland, a Professor at the UC Berkeley School of Information brought the life and the work of Emanuel Goldberg to light. Thanks to him, his works and innovations, who had disappeared from our cultural and scientific heritage, return to light after being erased during fifty years. Goldberg had published dozens of articles, obtained patents, developed cameras, microdots, movie cameras, and he designed what he called a "Statistical Machine", the first electronic document retrieval machine. In France, if this rediscovery was made known to the world of information science, it has not had the impact it deserved in the scientific world. Therefore it is time to reconstruct his career and his work, and to analyse the reasons why some attempted to erase definitively his name and memory. $\diamond$

\section{CONFLIT D'INTÉRÊTS}

L'auteur déclare n'avoir aucun conflit d'intérêts concernant les données publiées dans cet article.

\section{RéFÉRENCES}

1. Fayet-Scribe S. L'information en ligne. BBF $1995 ; n^{\circ} 5$ : 83-5 (en ligne : http://bbf.enssib.fr/consulter/bbf-1995-05-0083-012).

2. Gilgenkrantz $\mathrm{S}$. Grandeur et vicissitudes de deux grandes compagnies d'optique allemandes. II. La firme Leitz. Med Sci (Paris) 2011 ; 27 : 421- 4

3. Luther $\mathrm{R}$, Goldberg $\varepsilon$. Beitrage zur Kinetik photochemischer reaktionen. Jahrbuch für Photographie 1907 ; 21 : 283-4 (abstract).

4. Goldberg $\varepsilon$. Densographe, appareil enregistreur pour la mesure du noircissement des plaques photographiques. Bull Soc Fr Photog 1910 ; $n^{\circ} 10: 336$.

5. Goldberg $\varepsilon$. Sur la préparation de prismes gris-neutres et d'écran à teinte croissante pour la photométrie et la photographie. Rev Sci Photog $1911 ; 5$ : 248-53.

6. Ivens J, Destanque R. Joris Ivens ou la mémoire d'un regard. Paris : BFB, $1982: 340 \mathrm{p}$.

7. Buckland MK. The kinamo movie camera, Emanuel Goldberg and Joris Evens. Fim History $2008: 20: 49-58$.

8. Goldberg $\varepsilon$. Methods of photographic registration. BrJ Photog $1932 ; 79: 533-4$.

9. Buckland MK. Emanuel Goldberg (1881-1970) : pioneer of information science. http://www.sims.berkeley.edu/ Buckland/Goldberg. html

10. Buckland MK. Emanuel Goldberg and his knowledge machine. Wesport CT USA-London UK : Libraries Unlimited, 2006 : 380 p.

11. Gilgenkrantz $S$. Grandeur et vicissitudes de deux grandes entreprises d'optique allemandes. I. La firme Zeiss. Med Sci (Paris) 2011 ; 27 : 204-7.

12. Hoover JE. The enemy's masterpiece of espionage. Reader's Digest $1946 ; 48$ : $1-6$.

13. Clelland CT, Risca V, Bancroft C. Hiding messages in DNA microdots. Nature $1999 ; 399: 533-4$.

14. Bush Vannevar. As we may think. Atlantic Monthly $1945 ; 176: 1-8$.

15. Augier M. Hypertextualité. Construction du sens et représentation des connaissances. Université de Nice-Sofia Antipolis : Thèse de Sciences de l'information et de la communication, $2005: 624 \mathrm{p}$.

\section{TIRÉS À PART}

S. Gilgenkrantz

\section{Collection L'Actualité Chimique-Livres}

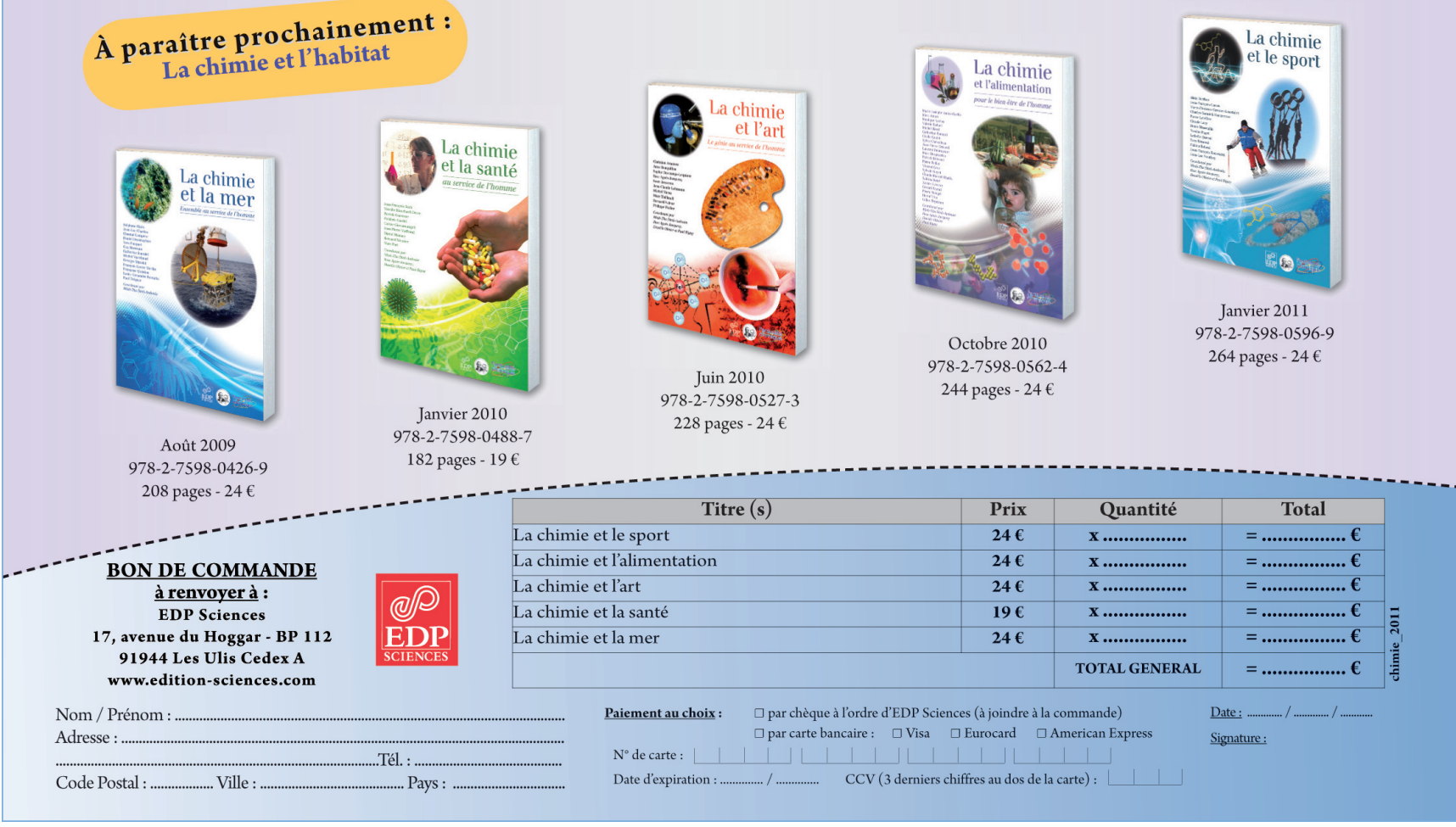

